\title{
Infinitesimally extremal Beltrami differentials of non-landslide type
}

\author{
GUOWU YAO
}

July 23, 2015

\begin{abstract}
In this paper, it is shown that there are infinitely many extremal Beltrami differentials of non-landside type and non-constant modulus in an infinitesimal equivalence class unless the class contains a unique extremal.
\end{abstract}

$\|$

\section{Introduction}

Let $S$ be a plane domain with at least two boundary points. The Teichmüller space $T(S)$ is the space of equivalence classes of quasiconformal maps $f$ from $S$ to a variable domain $f(S)$. Two quasiconformal maps $f$ from $S$ to $f(S)$ and $g$ from $S$ to $g(S)$ are equivalent if there is a conformal map $c$ from $f(S)$ onto $g(S)$ and a homotopy through quasiconformal maps $h_{t}$ mapping $S$ onto $g(S)$ such that $h_{0}=c \circ f, h_{1}=g$ and $h_{t}(p)=c \circ f(p)=g(p)$ for every $t \in[0,1]$ and every $p$ in the boundary of $S$. Denote by $[f]$ the Teichmüller equivalence class of $f$; also sometimes denote the equivalence class by $[\mu]$ where $\mu$ is the Beltrami differential of $f$.

Denote by $\operatorname{Bel}(S)$ the Banach space of Beltrami differentials $\mu=\mu(z) d \bar{z} / d z$ on $S$ with finite $L^{\infty}$-norm and by $M(S)$ the open unit ball in $\operatorname{Bel}(S)$.

For $\mu \in M(S)$, define

$$
k_{0}([\mu])=\inf \left\{\|\nu\|_{\infty}: \nu \in[\mu]\right\} .
$$

We say that $\mu$ is extremal in $[\mu]$ if $\|\mu\|_{\infty}=k_{0}([\mu])$, and uniquely extremal if $\|\nu\|_{\infty}>$ $k_{0}(\mu)$ for any other $\nu \in[\mu]$.

The cotangent space to $T(S)$ at the basepoint is the Banach space $Q(S)$ of integrable holomorphic quadratic differentials on $S$ with $L^{1}$-norm

$$
\|\varphi\|=\iint_{S}|\varphi(z)| d x d y<\infty .
$$

2010 Mathematics Subject Classification. Primary 30C75, 30C62.

Key words and phrases. Teichmüller space, infinitesimally extremal, non-landslide extremal.

The author was supported by the National Natural Science Foundation of China (Grant No. 11271216). 
In what follows, let $Q^{1}(S)$ denote the unit sphere of $Q(S)$. if

Two Beltrami differentials $\mu$ and $\nu$ in $\operatorname{Bel}(S)$ are said to be infinitesimally equivalent

$$
\iint_{S}(\mu-\nu) \varphi d x d y=0, \text { for any } \varphi \in Q(S) .
$$

The tangent space $Z(S)$ of $T(S)$ at the basepoint is defined as the quotient space of $\operatorname{Bel}(S)$ under the equivalence relation. Denote by $[\mu]_{Z}$ the equivalence class of $\mu$ in $Z(S)$. In particular, we use $\mathcal{N}(S)$ to denote the set of Beltrami differentials in $\operatorname{Bel}(S)$ that is equivalent to 0 .

$Z(S)$ is a Banach space and its standard sup-norm satisfies

$$
\left\|[\mu]_{Z}\right\|=\|\mu\|:=\sup _{\varphi \in Q^{1}(S)} \operatorname{Re} \iint_{S} \mu \varphi d x d y=\inf \left\{\|\nu\|_{\infty}: \nu \in[\mu]_{Z}\right\} .
$$

We say that $\mu$ is extremal (in $[\mu]_{Z}$ ) if $\|\mu\|_{\infty}=\left\|[\mu]_{Z}\right\|$, uniquely extremal if $\|\nu\|_{\infty}>\|\mu\|_{\infty}$ for any other $\nu \in[\mu]_{Z}$.

A Beltrami differential $\mu$ in $\operatorname{Bel}(S)$ is said to be of landslide type if there exists a non-empty open subset $E \subset S$ such that

$$
\underset{z \in E}{\operatorname{esssup}}|\mu(z)|<\|\mu\|_{\infty} ;
$$

otherwise, $\mu$ is said to be of non-landslide type.

The conception of "non-landslide" was firstly introduced by Li in [4 for extremal Beltrami differentials. Here, we generalize the definition for general case. In particular, a unique extremal is naturally of non-landslide type.

Let $\Delta$ be the unit disk in the complex plane. In [4, Z. Li investigated non-uniqueness of extremal Beltrami differentials of non-landslide type in a Teichmüller equivalence class in the universal Teichmüller space $T(\Delta)$ and proved the following theorem.

Theorem A. There is a point $[\mu]$ in $T(\Delta)$ such that $[\mu]$ contains infinitely many extremal Beltrami differentials of non-landslide type with non-constant modulus.

In 4, Li posed the following problem.

Problem. For any given $[\mu]$, is there always an extremal Beltrami differential $\widetilde{\mu}$ in $[\mu]$ which is of non-landslide type?

In [2], Fan answered the problem affirmatively and proved that if $[\mu]$ contains infinitely many extremals, then there always exist infinitely many extremals of nonlandslide type in $[\mu]$. The author gave a more precise formulation for the problem in [13. by use of variability set and point shift differentials, that is,

Theorem B. Let $[\mu]$ be given in $T(S)$. Then either each extremal in $[\mu]$ is of nonlandslide type or there are infinitely many non-landslide extremals of non-constant modulus in $[\mu]$.

The goal of this paper is to show a strengthened counterpart of Theorem $\mathrm{B}$ in the infinitesimal setting. 
Theorem 1. Let $[\mu]_{Z}$ be given in $Z(S)$. Then $[\mu]_{Z}$ contains infinitely many nonlandslide extremals of non-constant modulus unless $[\mu]_{Z}$ contains a unique extremal.

Unfortunately, due to the loss of the notion of boundary map and variability set, the required point shift differentials (see [9]) in the proof of Theorem B is no longer available. To overcome the difficulty, we develop a new technique in a self-contained way.

One must not expect that there always exists a non-landslide extremal of constant modulus in $[\mu]_{Z}$. The reason is that each extremal in $[\mu]_{Z}$ can be of non-constant modulus (see [1, 12]). Therefore, we have a direct corollary.

Corollary 1.1. There is a point $[\mu]_{Z}$ in $Z(S)$ such that $[\mu]_{Z}$ contains infinitely many non-landslide extremals and each non-landslide extremal in $[\mu]_{Z}$ is of non-constant modulus.

We will prove some lemmas in Section 2, Theorem 1 will be proved in Section 3. In addition, we define the local dilatation and discuss the landslide set for an infinitesimal equivalence class in the last section.

\section{Some lemmas}

Lemma 2.1. Let $\nu \in \operatorname{Bel}(\Delta)$. Then for any given $\epsilon>0$, there exists some $r \in(0,1)$ and $\mu \in \operatorname{Bel}(\Delta)$ such that

(1) $\mu \in \mathcal{N}(\Delta)$, (2) $\mu(z)=\nu(z), z \in \Delta_{r}$, (3) $\left\|\left.\mu\right|_{U_{r}}\right\|_{\infty}<\epsilon$, where $\Delta_{r}=\{z \in \Delta:|z|<r\}, r \in(0,1)$ and $U_{r}=\Delta \backslash \Delta_{r}$.

Proof. By Theorem 1.1 in [5], there exists a unique function $\beta(z)$, holomorphic in $\mathbb{C} \backslash \overline{\Delta_{r}}$, such that

$$
\mu(z)= \begin{cases}\nu(z), & z \in \Delta_{r}, \\ \beta(z), & z \in U_{r},\end{cases}
$$

belongs to $\mathcal{N}(\Delta)$; namely,

$$
\beta(z)=-\frac{z}{\pi\left(1-r^{2}\right)} \iint_{\Delta_{r}} \frac{\nu(z)}{\zeta-z} d \xi d \eta, z \in \mathbb{C} \backslash \overline{\Delta_{r}} .
$$

To complete the proof of this lemma, it is sufficient to show that $\left\|\left.\beta\right|_{U_{r}}\right\|_{\infty}<\epsilon$ for small $r>0$. We need to valuate $|\beta(z)|$ for $z \in U_{r}$. Let $z^{\prime}$ be the intersection point of the segment $\overline{o z}$ with the circle $\{|\zeta|=r\}$ and $B_{r}=\left\{\left|\zeta-z^{\prime}\right|<\frac{r}{2}\right\}$. Then for $z \in U_{r}$,

$$
\begin{aligned}
& |\beta(z)|=\frac{|z|}{\pi\left(1-r^{2}\right)}\left|\iint_{\Delta_{r}} \frac{\nu(z)}{\zeta-z} d \xi d \eta\right| \leq \frac{\|\nu\|_{\infty}}{\pi\left(1-r^{2}\right)} \iint_{\Delta_{r}} \frac{1}{|\zeta-z|} d \xi d \eta \\
& \leq M \iint_{\Delta_{r}} \frac{1}{\left|\zeta-z^{\prime}\right|} d \xi d \eta \leq M\left(\iint_{B_{r}} \frac{1}{\left|\zeta-z^{\prime}\right|} d \xi d \eta+\iint_{\Delta_{r} \backslash B_{r}} \frac{1}{\left|\zeta-z^{\prime}\right|} d \xi d \eta\right) \\
& \leq M\left(\pi r+\frac{2}{r} \iint_{\Delta_{r}} d \xi d \eta\right)=3 M \pi r=\frac{3 \pi\|\nu\|_{\infty} r}{\pi\left(1-r^{2}\right)},
\end{aligned}
$$

where $M=\frac{\|\nu\|_{\infty}}{\pi\left(1-r^{2}\right)}$. This lemma follows readily. 
Generally, for a given Beltrami differential $\mu \in \operatorname{Bel}(S)$ and a point $p \in S$, define

$$
\sigma(\mu, p)=\inf \left\{\operatorname{esssup}_{z \in U}|\mu(z)|: U \text { is an open neighborhood in } S \text { containing } p\right\}
$$

to be the local dilatation of $\mu$ at $p$. If $\sigma(\mu, p)=\|\mu\|_{\infty}$, we call $p$ a non-landslide point of $\mu$, otherwise, $p$ a landslide point of $\mu$. The collection of all landslide points of $\mu$ is called the landslide set of $\mu$, denoted by $L S(\mu)$. We call $N S L(\mu)=S \backslash L S(\mu)$ the non-landslide set of $\mu$. It is possible that $L S(\mu)=S$. It is obvious that $L S(\mu)$ is an open subset of $S$. In particular, $\mu$ is non-landslide if and only if $L S(\mu)=\emptyset$.

Remark 1. The local boundary dilatation of $\mu$ is defined for the boundary points (see Chapter 17 in [3]). Here, we generalize the notion for the inner points. Generally, $\sigma(\mu, p)$ and $|\mu(p)|$ are two different quantities for $p \in S$ and $\sigma(\mu, p) \geq|\mu(p)|$ for almost all $p \in S$ by Lebesgue's Theorem.

Throughout the paper, we denote by $\Delta(\zeta, r)$ the round disk $\{z:|z-\zeta|<r\}$ $(r>0)$.

Lemma 2.2. Suppose that $\mu \in \operatorname{Bel}(S)$ and $L S(\mu) \neq \emptyset$. Let $\alpha \in \operatorname{Bel}(S)$ with $\|\alpha\|_{\infty} \leq$ $\|\mu\|_{\infty}$. Then, for any $\zeta \in L S(\mu)$, there exists a disk $\Delta(\zeta, r)$ in $L S(\mu)$ and $\nu \in[\mu]_{Z}$ such that $\nu=\alpha$ on $\Delta(\zeta, r),\|\nu\|_{\infty}=\|\mu\|_{\infty}$ and $\nu(z)=\mu(z)$ on $N L S(\mu)$. In particular, $\nu$ vanishes on $\Delta(\zeta, r)$ when $\alpha=0$.

Proof. Choose sufficiently small $\rho>0$ such that the disk $D=\{|z-\zeta|<\rho\}$ is contained in $L S(\mu)$. Restrict $\mu$ on $D$. Then $\left\|\left.\mu\right|_{D}\right\|_{\infty}<\|\mu\|_{\infty}$. Let $\epsilon=\frac{\|\mu\|_{\infty}-\left\|\left.\mu\right|_{D}\right\|_{\infty}}{2}$. Applying Lemma 2.1 to $D$, we can find some small $r \in(0, \rho)$ and $\chi \in \mathcal{N}(D)$ such that $\chi=\alpha-\mu$ on $\Delta(\zeta, r)$ and $\|\chi\|_{\infty}<\epsilon$ on $D \backslash \Delta(\zeta, r)$. Put

$$
\nu(z)= \begin{cases}\chi(z)+\mu(z), & z \in D, \\ \mu(z), & z \in S \backslash D .\end{cases}
$$

Then $\nu \in[\mu]_{Z}$ and $\nu=\alpha$ on $\Delta(\zeta, r)$. It is clear that $\|\nu\|_{\infty}=\|\mu\|_{\infty}$ and $\nu(z)=\mu(z)$ on $N L S(\mu)$.

Lemma 2.3. Suppose $\mu \in \operatorname{Bel}(S)$ with $\|\mu\|_{\infty}=k$. Let $\mathscr{U}=\left\{\alpha \in[\mu]_{Z}:\|\alpha\|_{\infty}=k\right\}$. Then there exists a Beltrami differential $\nu \in \mathscr{U}$ such that $\nu(z)=0$ a.e. on $L S(\nu)$.

Proof. By Lemma 2.2, we can choose $\chi \in \mathscr{U}$ such that $N L S(\chi) \neq \emptyset$. If in addition $L S(\chi)=\emptyset$, then $\nu=\chi$ is the desired Beltrami differential. Otherwise, $L S(\chi)$ is a non-empty open subset in $S$. By Lemma 2.2, there exists a Beltrami differential $\eta \in \mathscr{U}$ such that

(1) $\eta(z)=\chi(z)$ on $N L S(\chi)$,

(2) $\eta(z)=0$ on some small disk $\Delta\left(z_{0}^{\prime}, r_{0}^{\prime}\right) \subset L S(\chi)$.

Let $\Lambda_{0}$ denote the collection of $\alpha \in \mathscr{U}$ with the following conditions:

(a) $\alpha(z)=\eta(z)$ on $N L S(\eta)$,

(b) there exists some small disk $\Delta(\zeta, r) \subset S$ such that $\alpha(z)=0$ on $\Delta(\zeta, r)$. 
It is obvious that $\eta \in \Lambda_{0}$. If $\alpha \in \Lambda_{0}$, let

$$
\rho_{0}(\alpha)=\sup \{r: \alpha(z)=0 \text { on some } \Delta(\zeta, r) \subset S\} .
$$

Put

$$
\rho_{0}=\sup \left\{\rho_{0}(\alpha): \alpha \in \Lambda_{0}\right\}
$$

We proceed with the construction of a sequence of Beltrami differentials $\left\{\chi_{n}\right\}$ in $\mathscr{U}$.

$n=0)$ Choose $\chi_{0} \in \Lambda_{0}$ such that $\rho_{0}\left(\chi_{0}\right) \geq r_{0}:=\frac{\rho_{0}}{2}$ and $\chi_{0}(z)=0$ on some $\Delta\left(z_{0}, r_{0}\right) \subset S$. If $\chi_{0}(z)=0$ a.e. on $L S\left(\chi_{0}\right)$, then $\nu=\chi_{0}$ is the desired Beltrami differential. Otherwise, by the definition of local dilatation there exists $z_{1}^{\prime} \in L S\left(\chi_{0}\right)$ such that $\sigma\left(\chi_{0}, z_{1}^{\prime}\right) \in(0, k)$. Again by Lemma 2.2. there exists a Beltrami differential $\eta_{0} \in \Lambda_{0}$ such that

(1) $\eta_{0}(z)=\chi_{0}(z)$ on $N L S\left(\chi_{0}\right)$,

(2) $\eta_{0}(z)=0$ on $\Delta\left(z_{0}, r_{0}\right)$,

(3) $\eta_{0}(z)=0$ on some small disk $\Delta\left(z_{1}^{\prime}, r_{1}^{\prime}\right) \subset L S\left(\chi_{0}\right) \backslash \Delta\left(z_{0}, r_{0}\right)$.

Let $\Lambda_{1}$ denote the collection of $\alpha \in \Lambda_{0}$ with the following conditions:

(a) $\alpha(z)=\eta_{0}(z)$ on $N L S\left(\eta_{0}\right)$,

(b) $\alpha(z)=0$ on $\Delta\left(z_{0}, r_{0}\right)$,

(c) there exists some small disk $\Delta(\zeta, r) \subset S \backslash \Delta\left(z_{0}, r_{0}\right)$ such that $\alpha(z)=0$ on $\Delta(\zeta, r)$.

It is obvious that $\eta_{0} \in \Lambda_{1}$. If $\alpha \in \Lambda_{1}$, let

$$
\rho_{1}(\alpha)=\sup \left\{r: \alpha(z)=0 \text { on some } \Delta(\zeta, r) \subset S \backslash \Delta\left(z_{0}, r_{0}\right)\right\} .
$$

Put

$$
\rho_{1}=\sup \left\{\rho_{1}(\alpha): \alpha \in \Lambda_{1}\right\} .
$$

$n=1)$ Choose $\chi_{1} \in \Lambda_{1}$ such that $\rho_{1}\left(\chi_{1}\right) \geq r_{1}:=\frac{\rho_{1}}{2}$ and $\chi_{1}(z)=0$ on some $\Delta\left(z_{1}, r_{1}\right) \subset S$. If $\chi_{1}(z)=0$ a.e. on $L S\left(\chi_{1}\right)$, then $\nu=\chi_{1}$ is the desired Beltrami differential. Otherwise, by the definition of local dilatation there exists $z_{2}^{\prime} \in L S\left(\chi_{1}\right)$ such that $\sigma\left(\chi_{1}, z_{2}^{\prime}\right) \in(0, k)$. Again by Lemma 2.2. there exists a Beltrami differential $\eta_{1} \in \Lambda_{1}$ such that

(1) $\eta_{1}(z)=\chi_{1}(z)$ on $N L S\left(\chi_{1}\right)$,

(2) $\eta_{1}(z)=0$ on $\Delta\left(z_{0}, r_{0}\right) \cup \Delta\left(z_{1}, r_{1}\right)$,

(3) $\eta_{1}(z)=0$ on some small disk $\Delta\left(z_{2}^{\prime}, r_{2}^{\prime}\right) \subset L S\left(\chi_{1}\right) \backslash\left(\Delta\left(z_{0}, r_{0}\right) \cup \Delta\left(z_{1}, r_{1}\right)\right)$.

Let $\Lambda_{2}$ denote the collection of $\alpha \in \Lambda_{1}$ with the following conditions:

(a) $\alpha(z)=\eta_{1}(z)$ on $N L S\left(\eta_{1}\right)$,

(b) $\alpha(z)=0$ on $\Delta\left(z_{0}, r_{0}\right) \cup \Delta\left(z_{1}, r_{1}\right)$,

(c) there exists some small disk $\Delta(\zeta, r) \subset S \backslash\left(\Delta\left(z_{0}, r_{0}\right) \cup \Delta\left(z_{1}, r_{1}\right)\right)$ such that $\alpha(z)=0$ on $\Delta(\zeta, r)$.

It is obvious that $\eta_{1} \in \Lambda_{2}$. If $\alpha \in \Lambda_{2}$, let

$$
\rho_{2}(\alpha)=\sup \left\{r: \alpha(z)=0 \text { on some } \Delta(\zeta, r) \subset S \backslash\left(\Delta\left(z_{0}, r_{0}\right) \cup \Delta\left(z_{1}, r_{1}\right)\right)\right\} .
$$

Put

$$
\rho_{2}=\sup \left\{\rho_{2}(\alpha): \alpha \in \Lambda_{2}\right\} .
$$


$n \rightarrow n+1)$ If we can choose a $\chi_{n} \in \Lambda_{n}$ such that $\chi_{n}(z)=0$ a.e. on $L S\left(\chi_{n}\right)$, then let $\nu=\chi_{n}$. Otherwise, as the previous, we can find an $\eta_{n} \in \Lambda_{n}$ such that

(1) $\eta_{n}(z)=\chi_{n}(z)$ on $N L S\left(\chi_{n}\right)$,

(2) $\eta(z)=0$ on $\left.\bigcup_{k=0}^{n-1} \Delta\left(z_{k}, r_{k}\right)\right)$,

(3) $\eta_{n}(z)=0$ on some small disk $\Delta\left(z_{n+1}^{\prime}, r_{n+1}^{\prime}\right) \subset L S\left(\chi_{n}\right) \backslash\left(\bigcup_{k=0}^{n-1} \Delta\left(z_{k}, r_{k}\right)\right)$.

Proceeding as above, we find five sequences, $\left\{\chi_{n} \in \Lambda_{n}\right\},\left\{\eta_{n} \in \Lambda_{n}\right\},\left\{\Lambda_{n} \subset \mathscr{U}\right\}$, $\left\{\rho_{n}\right\},\left\{\Delta\left(z_{n}, r_{n}\right) \subset S\right\}\left(r_{n}=\frac{\rho_{n}}{2}\right)$ as follows.

$\chi_{n} \in \Lambda_{n}$ satisfies $\rho_{n}\left(\chi_{n}\right) \geq r_{n}:=\frac{\rho_{n}}{2}$ and

$$
\chi_{n}(z)=0 \text { on some } \Delta\left(z_{n}, r_{n}\right) \subset S \backslash\left(\bigcup_{k=0}^{n-1} \Delta\left(z_{k}, r_{k}\right)\right) .
$$

$\Lambda_{n+1}$ is the collection of $\alpha \in \Lambda_{n}$ with the following conditions:

(a) $\alpha(z)=\eta_{n}(z)$ on $N L S\left(\eta_{n}\right)$,

(b) $\alpha(z)=0$ on $\left.\bigcup_{k=0}^{n} \Delta\left(z_{k}, r_{k}\right)\right)$,

(c) there exists some small disk $\Delta(\zeta, r) \subset S \backslash\left(\bigcup_{k=0}^{n} \Delta\left(z_{k}, r_{k}\right)\right)$ such that $\alpha(z)=0$ on $\Delta(\zeta, r)$.

It is obvious that $\eta_{n} \in \Lambda_{n+1}$. If $\alpha \in \Lambda_{n+1}$, let

$$
\rho_{n+1}(\alpha)=\sup \left\{r: \alpha(z)=0 \text { on } \Delta(\zeta, r) \subset S \backslash\left(\bigcup_{k=0}^{n} \Delta\left(z_{k}, r_{k}\right)\right)\right\} .
$$

Put

$$
\rho_{n+1}=\sup \left\{\rho_{n+1}(\alpha): \alpha \in \Lambda_{n+1}\right\} .
$$

It is clear that

$$
\lim _{n \rightarrow \infty} \rho_{n}=\lim _{n \rightarrow \infty} r_{n}=0 .
$$

Let $B_{n}=\bigcup_{k=0}^{n} \Delta\left(z_{k}, r_{k}\right), n=0,1, \cdots$. Then

$$
B=\lim _{n \rightarrow \infty} B_{n}=\bigcup_{n=0}^{\infty} \Delta\left(z_{n}, r_{n}\right) \subset S .
$$

By the *-weak compactness, there exists a subsequence of $\left\{\chi_{n}\right\}$, still denoted by $\left\{\chi_{n}\right\}$, which converges to a limit $\nu \in \operatorname{Bel}(S)$ in the *-weak topology, that is, for any $\phi \in L^{1}(S)$,

$$
\lim _{n \rightarrow \infty} \iint_{S} \chi_{n}(z) \phi(z) d x d y=\iint_{S} \nu(z) \phi(z) d x d y .
$$

When $\phi \in Q(S)$, since $\chi_{n} \in[\mu]_{Z}$, we have

$$
\iint_{S} \chi_{n}(z) \phi(z) d x d y=\iint_{S} \mu(z) \phi(z) d x d y \text { for all } n .
$$

Therefore,

$$
\iint_{S} \nu(z) \phi(z) d x d y=\iint_{S} \mu(z) \phi(z) d x d y
$$


for all $\phi \in Q(S)$ and hence $\nu \in[\mu]_{Z}$. On the other hand, since $\chi_{n}$ converges to $\nu$ in the *-weak topology, it follows by the standard functional analysis theory that

$$
\|\nu\|_{\infty} \leq \liminf _{n \rightarrow \infty}\left\|\chi_{n}\right\|_{\infty}=k .
$$

Claim. $\nu \in \mathscr{U}$ and $\nu(z)=0$ a.e. on $L S(\nu)$.

Firstly, by the inductive construction, we see that $\chi_{n+1}(z)=\chi_{n}(z)$ on $N L S\left(\chi_{n}\right)$. So it holds that $\nu(z)=\chi_{n}(z)$ on $N L S\left(\chi_{n}\right)$ for all $n$. Since $N L S\left(\chi_{n}\right) \supseteq N L S(\chi)$, we have $\|\nu\|_{\infty}=k$ and hence $\nu \in \mathscr{U}$.

Secondly, since $\chi_{n}(z)=0$ on $B_{n}$, we find that $\chi_{n}(z) \rightarrow 0$ for all $z \in B$ as $n \rightarrow \infty$. Therefore, by the uniqueness of *-weak limit, we must have $\nu(z)=0$ on $B$.

Now we show that $\nu(z)=0$ a.e. on $L S(\nu)$. Suppose to the contrary. Then there exists a point $p \in L S(\nu)$ such that $\sigma(p, \nu) \in(0, k)$. Applying Lemma 2.2. we can find a Beltrami differential $\nu^{\prime} \in \mathscr{U}$ such that

(1) $\nu^{\prime}(z)=\nu(z)$ on $N L S(\nu)$,

(2) $\nu^{\prime}(z)=0$ on $B$,

(3) $\nu^{\prime}(z)=0$ on some small disk $\Delta(p, r) \subset S \backslash B$.

It is obvious that $\nu^{\prime}$ belongs to $\bigcap_{n=0}^{\infty} \Lambda_{n}$. However, by (2.1) it contradicts the choice of $\chi_{n}$. The claim is proved, so is the lemma.

\section{Proof of Theorem 1}

Lemma 3.1. Let $S=\Delta$. Fix $k>0$. Then (1) there exist infinitely many Beltrami differentials $\mu \in[0]_{Z}$ such that each $\mu$ is non-landslide and has a constant modulus $k$; (2) there exist infinitely many Beltrami differentials $\mu \in[0]_{Z}$ such that each $\mu$ is non-landslide with $\|\mu\|_{\infty}=k$ but $\mu$ has not a constant modulus.

Proof. (1) Let $\mu_{n}(z)=k \frac{z^{n}}{\left.z\right|^{n}}$ for integer number $n \geq 1$. It is easy to verify that $\mu_{n} \in[0]_{Z}$. It is obvious that each $\mu_{n}$ is non-landslide and has a constant modulus $k$.

(2) Let $E \subset \Delta$ be a compact subset with positive measure and empty interior. Since $\Delta \backslash E$ is a non-empty open subset of $\Delta$, we can choose two sequences $\left\{p_{n}\right\}$ in $\Delta \backslash E$ and $\left\{r_{n}\right\}$ in $(0,1)$ respectively such that

$$
\Delta \backslash E=A \bigcup_{n=1}^{\infty} B_{r_{n}}\left(p_{n}\right) \text { and } B_{r_{i}}\left(p_{i}\right) \bigcap B_{r_{j}}\left(p_{j}\right)=\emptyset, i \neq j,
$$

where $A$ is a set of measure 0 and $B_{r_{n}}\left(p_{n}\right):=B_{n}$ denotes the round disk centered at $p_{n}$ with radius $r_{n}$. Keep $\mu(z)=0$ on $E$. We apply (1) on $B_{n}$ and then obtain infinitely many $\eta$ which are infinitesimally equivalent to $\left.0\right|_{B_{n}}$ on $B_{n}$ with modulus $|\eta(z)| \equiv k$ on $B_{n}$. Modify $\left.0\right|_{B_{n}}$ on every $B_{n}$ in such an equivalent way. Then we can find infinitely many Beltrami differentials $\mu \in[0]_{Z}$ such that each $\mu$ is non-landslide with $\|\mu\|_{\infty}=k$ but $\mu$ has not a constant modulus since $\mu(z) \equiv 0$ on $E$. 
Proof of Theorem 1, Assume that the extremal in $[\mu]_{Z}$ is not unique. We show that $[\mu]_{Z}$ contains infinitely many extremal Beltrami differentials of non-landslide type.

Case 1. $\mu$ contains an extremal of landslide type.

By Lemma 2.3, there is an extremal Beltrami differential in $[\mu]_{Z}$, say $\mu$, such that $L S(\mu) \neq \emptyset$ and $\mu(z)=0$ on $L S(\mu)$. We start from $\mu$ to produce infinitely many extremal Beltrami differentials of non-landslide type in $[\mu]_{Z}$.

Since $L S(\mu)$ is a non-empty open subset of $S$, we can choose two sequences $\left\{p_{n}\right\}$ in $L S(\mu)$ and $\left\{r_{n}\right\}$ in $(0,1)$ respectively such that

$$
L S(\mu)=A \bigcup_{n=1}^{\infty} B_{r_{n}}\left(p_{n}\right) \text { and } B_{r_{i}}\left(p_{i}\right) \bigcap B_{r_{j}}\left(p_{j}\right)=\emptyset, i \neq j,
$$

where $A$ is a set of measure 0 and $B_{r_{n}}\left(p_{n}\right):=B_{n}$ denotes the round disk centered at $p_{n}$ with radius $r_{n}$. Notice that $\left[\left.\mu\right|_{B_{n}}\right]_{Z}=\left[\left.0\right|_{B_{n}}\right]_{Z}$ on every $B_{n}$ where we regard $\left[\left.\mu\right|_{B_{n}}\right]_{Z}$ as a point in the space $Z\left(B_{n}\right)$. We apply Lemma 3.1 on $B_{n}$ and then find $\eta \in\left[\left.\mu\right|_{B_{n}}\right]_{Z}$ with modulus $|\eta(z)| \equiv\left\|[\mu]_{Z}\right\|$ on $B_{n}$. Modifying $\mu$ on every $B_{n}$ in such an equivalent way, we then get infinitely many non-landslide extremals in $[\mu]_{Z}$.

To obtain infinitely many non-landslide extremals of non-constant modulus in $[\mu]_{Z}$, we only need to modify $\left.\mu\right|_{B}$ into $\left.\eta\right|_{B}$ on some disk $B$ in $L S(\mu)$, say $B=B_{1}$ such that $\left.\eta\right|_{B}$ is of non-landslide type and non-constant modulus with respect to the quantity $\left\|[\mu]_{Z}\right\|$. This can be done by applying Lemma 3.1 (2).

Case 2. Each extremal in $[\mu]_{Z}$ is of non-landslide type (see Remark 2).

If all extremals in $[\mu]_{Z}$ are of non-constant modulus, the proof is a fortiori. Assume, that $[\mu]_{Z}$ contains at least an extremal of constant modulus, say $\nu$. Generally, it is known [7, 10] that $[\mu]_{Z}$ contains at least an extremal of non-constant modulus unless $[\mu]_{Z}$ contains a unique extremal of constant modulus. Say, $\mu$ is an extremal of nonconstant modulus. Consider the Beltrami differentials curve $\eta_{t}=t \mu+(1-t) \nu, t \in[0,1]$. Then, $\eta_{t} \in[\mu]_{Z}$ and $\left|\eta_{t}(z)\right| \leq t|\mu(z)|+(1-t)|\nu(z)| \leq\left\|[\mu]_{Z}\right\|$ for all $t \in[0,1]$ and almost all $z \in S$. Hence every $\eta_{t}$ is extremal in $[\mu]_{Z}$. It is clear that $\eta_{t} \neq \eta_{s}$ if $t \neq s$ in $[0,1]$. Moreover, for every $t \in(0,1), \eta_{t}$ is non-landslide and of non-constant modulus. The concludes the proof of the theorem.

Remark 2. It is an open problem whether there exists $[\mu]_{Z}$ such that the extremal in $[\mu]_{Z}$ is not unique and each extremal in $[\mu]_{Z}$ is non-landslide.

\section{On landslide set of an infinitesimal class}

Let $\mu \in \operatorname{Bel}(S)$. Define the quantity

$$
\sigma\left([\mu]_{Z}, p\right)=\inf \left\{\sigma(\eta, p): \eta \text { is an extremal in }[\mu]_{Z}\right\}
$$

to be the local dilatation of $[\mu]_{Z}$ at $p \in S$. If $\sigma\left([\mu]_{Z}, p\right)=\|\mu\|$, we call $p$ a non-landslide point of $[\mu]_{Z}$; otherwise $p$ a landslide point of $[\mu]_{Z}$. The collection of all landslide points of $[\mu]_{Z}$ is called the landslide set of $[\mu]_{Z}$, denoted by $L S\left([\mu]_{Z}\right) . L S\left([\mu]_{Z}\right)$ is clearly an open subset in $S$.

Proposition 4.1. Let $[\mu]_{Z} \in Z(S)$. Then for any $p \in S$, we have either $\sigma\left([\mu]_{Z}, p\right)=0$ or $\sigma\left([\mu]_{Z}, p\right)=\left\|[\mu]_{Z}\right\|$. 
Proof. Suppose $\sigma\left([\mu]_{Z}, p\right)<\|\mu\|$. Then there exists some extremal in $[\mu]$, say $\mu$, and some small disk $B_{r}$ in $S$ with center $p$ and radius $r$ such that $\operatorname{esssup}_{z \in B_{r}}|\mu(z)|<\|\mu\|$. Restricted on $B_{r}$, there exists some $\eta \in\left[\left.\mu\right|_{B_{r}}\right]$ such that $\|\eta\|_{\infty}<\|\mu\|$ and $\eta(z) \equiv 0$ on some small disk $B_{\delta}$ in $B_{r}$ with center $p$ and radius $\delta<r$ by Lemma 2.2. This gives $\sigma\left([\mu]_{Z}, p\right)=0$.

A Beltrami differential $\mu$ is called to be locally extremal if for any subdomain $G \subset S$, it is extremal in its class in $Z(G)$. The notion of locally extremal was first introduced by Sheretov in 8 .

The following three conditions are equivalent:

(i) all extremals in $[\mu]_{Z}$ are locally extremal;

(ii) each extremal in $[\mu]_{Z}$ is non-landslide;

(iii) $\sigma\left([\mu]_{Z}, p\right)=\|\mu\|$ for all $p \in S$, i.e. $L S\left([\mu]_{Z}\right)=\emptyset$.

There is no essential relation among local extremals and non-landslide extremals. In fact, a local extremal can be of landslide type due to the construction in Theorem 1 of [11. The example of a local extremal (not a unique extremal) of constant modulus was first given by Reich in [6].

The following theorem says that every component of $L S\left([\mu]_{Z}\right)$ has no holes.

Theorem 2. Let $[\mu]_{Z} \in Z(S)$. Then every component of $L S\left([\mu]_{Z}\right)$ is simply-connected.

Proof. If $L S\left([\mu]_{Z}\right)=\emptyset$, the theorem is a fortiori. Suppose $L S\left([\mu]_{Z}\right) \neq \emptyset$. Assume, by contradiction, that some component $D$ of $L S\left([\mu]_{Z}\right)$ has holes. Since $L S\left([\mu]_{Z}\right)$ is open, we can choose a Jordan curve $C$ in $D$ such that the Jordan domain $J$ contoured by $C$ is contained in $S$ and there is at least a hole denoted by $H$ located in $J$. Notice that the hole $H$ is actually a connected component of $S \backslash L S\left([\mu]_{Z}\right)$.

For any point $p \in C$, by Proposition 4.1 , there exists some extremal $\nu_{p}$ in $[\mu]_{Z}$ such that $\nu_{p}(z) \equiv 0$ on a small disk $B_{r_{p}}(p)$ in $D$ with center $p$ and radius $r_{p}$. When $p$ varies along $C$, all such $B_{r_{p}}(p)$ cover the curve $c$. By the finite covering theorem, we can choose finite disks $B_{r_{p_{j}}}\left(p_{j}\right)(j=1,2 \cdots, n)$ covering $C$. Let $\nu_{p_{j}}(j=1,2 \cdots, n)$ be these corresponding extremals. Put

$$
\eta=\frac{\nu_{p_{1}}+\nu_{p_{2}}+\cdots+\nu_{p_{n}}}{n} .
$$

Then $\eta \in[\mu]_{Z}$ and $\eta$ is an extremal. In particular, we see that $\sigma(\eta, p) \leq \frac{n-1}{n}\left\|[\mu]_{Z}\right\|$ for all points $p$ in $R=\bigcup_{j=1}^{n} B_{r_{p_{j}}}\left(p_{j}\right)$. Set $\widetilde{J}=J \bigcup R$. Then $\widetilde{J}$ is also a Jordan domain in $S$. Now, one easily shows that $\eta$ is not extremal restricted on $\widetilde{J}$ and hence $\sigma(\eta, p)<\left\|[\mu]_{Z}\right\|$ for all $p \in \widetilde{J}$. Whereas, for $p \in H$, we have $\sigma\left([\mu]_{Z}, p\right)=\left\|[\mu]_{Z}\right\|$, which is a desired contradiction.

\section{REFERENCES}

[1] V. Božin, N. Lakic and V. Marković and M. Mateljević, Unique extremality, J. Anal. Math. 75 (1998), 299-338. 
[2] J. Fan, On extremal quasiconformal mappings of non-landslide type, Proc. Amer. Math. Soc. 139 (2011), no. 8, 2729-2733.

[3] F. P. Gardiner and N. Lakic, Quasiconformal Teichmüller Theory, Mathematical Surveys and Monographs, Vol. 76, Amer. Math. Soc. Providence, RI, 2000.

[4] Z. Li, A note on extremal quasiconformal mappings, Sci. China, Ser. A, 53 (2010), 63-70.

[5] E. Reich, An extremum problem for analytic functions with area norm, Ann. Acad. Sci. Fenn. Ser. A. I. Math. 2 (1976), 429-445.

[6] E. Reich, On the uniqueness question for Hahn-Banach extensions from the space of $L^{1}$ analytic functions, Proc. Amer. Math. Soc. 88 (1983), 305-310.

[7] E. Reich, Non-uniquely extremal quasiconformal mappings, Libertas Mathematica, 20 (2000), 33-38.

[8] V. G. Sheretov, Locally extremal quasiconformal mappings, Soviet Math. Dokl. 21 (1980), 343,345.

[9] K. Strebel, Point shift differentials and extremal quasiconformal mappings, Ann. Acad. Sci. Fenn. Math. 23 (1998), 475-494.

[10] G. W. Yao and Y. Qi, On the modulus of extremal Beltrami coefficients, J. Math. Kyoto Univ. 46 (2006), 235-247.

[11] G. W. Yao, Unique extremality, local extremality and extremal non-decreasable dilatations, Bull. Austral. Math. Soc. 75 (2007), 321-329.

[12] G. W. Yao, Existence of extremal Beltrami coefficients with non-constant modulus, Nagoya Math. J. 199 (2010), 1-14.

[13] G. W. Yao, Extremal Beltrami differentials of non-landslide type, Proc. Edinb. Math. Soc. to appear.

Guowu Yao

Department of Mathematical Sciences

Tsinghua University

Beijing, 100084, People's Republic of China

E-mail: gwyao@math.tsinghua.edu.cn 